\title{
Gestational diabetes: costs and consequences
}

\author{
R. S. Lindsay
}

Received: 27 September 2010 / Accepted: 8 October 2010 /Published online: 26 November 2010

(C) Springer-Verlag 2010

\begin{abstract}
Important new evidence regarding the relationships of maternal glucose to fetal growth and pregnancy complications have emerged in the last 5 years. Major international efforts are underway to translate this evidence into clinical practice and create international norms for the diagnosis of gestational diabetes. Consideration of the costs and benefits of the screening, diagnosis and treatment of gestational diabetes is complex but likely to be central to this translational programme.
\end{abstract}

Keywords Gestational diabetes $\cdot$ HAPO $\cdot$ Hyperglycaemia and Pregnancy Outcome study. Pregnancy

\begin{tabular}{ll}
\multicolumn{2}{l}{ Abbreviations } \\
ACHOIS & $\begin{array}{l}\text { Australian Carbohydrate Intolerance Study in } \\
\text { Pregnant Women }\end{array}$ \\
GDM & $\begin{array}{l}\text { Gestational diabetes mellitus } \\
\text { HyPO }\end{array}$ \\
HTA & $\begin{array}{l}\text { Health Technology Assessment } \\
\text { International Association of Diabetes and }\end{array}$ \\
IADPSG & $\begin{array}{l}\text { Pregnancy Study Groups } \\
\text { Large for gestational age }\end{array}$ \\
LGA & $\begin{array}{l}\text { National Institute for Health and Clinical } \\
\text { NICE }\end{array}$ \\
QALCellence \\
MFMU & $\begin{array}{l}\text { Quality-adjusted life-years } \\
\text { Maternal Fetal Medicines Unit Network }\end{array}$
\end{tabular}

There have been longstanding controversies over diagnostic criteria for gestational diabetes mellitus (GDM) and

R. S. Lindsay $(\bowtie)$

BHF Glasgow Cardiovascular Research Centre,

Faculty of Medicine, University of Glasgow,

Glasgow G12 8AT, UK

e-mail: r.lindsay@clinmed.gla.ac.uk whether screening itself is appropriate. The international literature has been difficult to reconcile owing to very different strategies for screening and diagnosis in different countries. In 2002 the National Institute for Health and Clinical Excellence (NICE) Health Technology Assessment (HTA) concluded that there was insufficient evidence to advocate universal screening for gestational diabetes [1]. At the same time the NICE HTA also stated that there were clearly some women in whom maternal hyperglycaemia was causing adverse fetal outcomes. There have been important developments in the evidence base since this report, which have led to a recent urgent update of the HTA [2]. In 2008 the multinational Hyperglycaemia and Pregnancy Outcome (HAPO) study [3] examined associations between maternal glucose tolerance and neonatal outcomes in an unprecedentedly large sample of over 23,000 women. The study showed a continuous relationship of maternal glucose to birthweight and macrosomia. Two major trials examining the effect on pregnancy outcomes of management of GDM were published in 2005 and 2009, showing a reduction of adverse outcomes with detection and treatment. These were the Australian Carbohydrate Intolerance Study in Pregnant Women (ACHOIS) [4] and the Maternal Fetal Medicines Unit Network (MFMU) [5], respectively. A randomised controlled trial showed the efficacy of metformin [6], adding to the older literature on the use of glibenclamide (known as glyburide in the USA and Canada) [7] in GDM.

These major trials represent important advances in the field and are very welcome. They also introduce some complex issues as to how best this evidence translates into healthcare. In this context the analysis of the cost-utility of screening for GDM by Round et al. in this issue of Diabetologia is timely and important [8]. 
Central to all of the problems of the definition of GDM is the continuous relationship of maternal glucose and neonatal outcomes, now clearly defined in HAPO but also detected in older studies $[9,10]$. As maternal glucose rises, the risk of macrosomia and other complications increases. This continuum of risk creates potential for opinion and debate on the level of maternal blood glucose at which GDM should be diagnosed. Furthermore, one can debate whether this set point should be determined by clinical risk (now available from the HAPO sample), measures of clinical effectiveness or cost effectiveness.

If diagnostic criteria are to be rationalised internationally then consensus will be necessary. One organised approach to this has been the initiative of the International Association of Diabetes and Pregnancy Study Groups (IADPSG) to use the HAPO data to reconsider screening for and diagnosis of GDM, with their opinions presented as a consensus report published in March 2010 [11]. In their analysis the IADPSG set cut-off points for GDM where key outcomes in the HAPO study (birthweight, cord C-peptide level, per cent body fat above the 90th percentile) were increased by 1.75 fold compared with women with mean glucose concentrations for the population [11]. A controversial result of this approach is the frequency of the diagnosis potentially increasing to $16-20 \%$ of pregnant women. This rate appears unacceptably high to many clinicians and would represent a radical redefinition of the diagnosis in many countries.

Round et al. base their approach on the calculation of a woman's theoretical risk of GDM and improvements in adverse outcomes using data from the two major intervention studies. Critically, the decisions of whether to screen and which method to use is based on individual risk of GDM, with different strategies being considered as costeffective depending on the woman's underlying risk.

This is an attractive approach. Nevertheless, controversies remain. Unsurprisingly, one issue is the definition of GDM. Within the confines of this important modelling exercise the effectiveness of treatment relates to GDM as defined within the ACHOIS and MFMU studies. Notably, the diagnoses are different between the studies and both studies deliberately excluded (as did HAPO) some of the women with the highest level of blood glucose. This was for ethical reasons, as both studies attempted to limit treatment in the control limbs. In the ACHOIS trial, women with a fasting glucose level of $\geq 7.8 \mathrm{mmol} / 1$ or a $2 \mathrm{~h}$ glucose level of $\geq 11.1 \mathrm{mmol} / 1$ were excluded. The MFMU trial was overtly designed to assess only 'mild' gestational diabetes, and women with a fasting glucose level of $\geq 5.3 \mathrm{mmol} /$ 1 were excluded. Overall, the ACHOIS trial appears to have included mothers with higher levels of blood glucose, on average, than the MFMU, with the proportion of large for gestational age (LGA) babies - defined as birthweight over the 90th percentile adjusted for gestational age and sex-in the control group being $22 \%$ vs $14.5 \%$, respectively. Perhaps in keeping with this sampling of different parts of the spectrum of maternal glucose, differences in outcomes between groups in the ACHOIS trial were more dramatic. Serious perinatal outcomes (a composite of death, shoulder dystocia, bone fracture and nerve palsy) fell from $4 \%$ to $1 \%$, and reductions in birthweight (by $147 \mathrm{~g}$ ), macrosomia (weight $>4 \mathrm{~kg}$ at birth) and LGA [4] were also observed. By contrast, the primary outcome measure (a composite of perinatal mortality, hypoglycaemia, hyperbilirubinaemia, neonatal hyperinsulinaemia and birth trauma) was not significantly reduced in the MFMU study, but there were significant reductions in mean birthweight (by $106 \mathrm{~g}$ ), macrosomia, LGA and shoulder dystocia in the MFMU study. There were also other maternal benefits. A reduction in pre-eclampsia narrowly failed to achieve significance with treatment of GDM in the ACHOIS trial and were significant in the MFMU study. The proportion of Caesarean sections $(26.9 \%$ in the intervention group vs $33.8 \%$ in the control group) was lower in the intervention group than in the control group in the MFMU study [5], and mothers gained less weight with intervention in both trials $[4,5]$.

It is unsurprising that the benefits observed differ between studies, as the part of the population of pregnant women sampled was likely different. Further, diagnosis and treatment occurred within the routine care groups, at least in the ACHOIS study, where some $3 \%$ of women in the routine care group were prescribed insulin. A much smaller proportion of control women were similarly treated in the MFMU study. This would be expected to reduce the difference in clinical outcomes between groups and lead to an underestimate of the benefit of intervention. Conversely, and as discussed by Round et al. [8], both studies entirely or predominantly involved women who had already 'failed' a stimulatory screening test and as such may be a more glucose intolerant population than those diagnosed with OGTT alone. Taken together, the definition of the effect of treatment-critical to measures of clinical and cost-effectiveness-becomes difficult.

The importance of the part of the population assessed and the underlying risk is highlighted in the informative sensitivity analysis performed by Round et al. [8]. The key issue is the estimate of the improvement in outcomes (and therefore gains in quality-adjusted life-years [QALY]) in the two studies. Thus, while analysis of the data of the two trials combined suggests that women with an individual GDM risk of $<1 \%$ should not undergo routine screening, this falls to $0.6 \%$ when based on the ACHOIS data and rises to $4 \%$ if outcomes from the MFMU trial are used. Clearly, each model would lead to quite different clinical guidance. One of the important factors driving the QALY 
gains is avoidance of perinatal deaths. Such analyses can also be criticised, since the number of perinatal deaths in the control limb of the ACHOIS trial was low and small changes in this rate could mark major changes in the policies for screening - an issue also explored in the sensitivity analyses published as part of the NICE diabetes and pregnancy report [12].

Despite these uncertainties, it is critical that the data from these large trials are translated into clinical practice. Pragmatically, now that we have the ACHOIS and MFMU trials, it will be difficult to design studies ethically with as little intervention in the control limb. The measures of effect that we have now may not be substantially improved. An important next step in these analyses will be translation of their recommendations into population models. The obvious resource for these efforts would be the HAPO dataset and it is hoped that such analyses will be supported.

A final point should be stressed. A further abiding message of the two intervention trials is that outcomes were improved by relatively non-invasive strategies. The proportion of women for whom management of GDM involved exogenous insulin was only $8 \%$ in the MFMU study [5] and $20 \%$ in the ACHOIS study [4]. The great majority of women with GDM were effectively managed by diet, lifestyle and glucose monitoring alone. Neither of the intervention trials used the oral hypoglycaemic agents metformin or glibenclamide and their use should further reduce costs. Furthermore, the group of pregnant women defined by the IADPSG criteria, although large, has a proportion of LGA babies intermediate between the two intervention studies $(16.2 \%$ when the criteria are applied to the HAPO population). This suggests that the benefits seen in the intervention studies might indeed be translated into practice even in the relatively large part of the population defined by the IADPSG criteria. Cost-effectiveness may become key in our consideration of who should be screened for GDM and indeed may potentially be considered in defining that part of the population formally diagnosed as having GDM. If so, it will be incumbent on the clinical and research community to develop programmes to deliver care more cost-effectively to this potentially large group of women so that these benefits can be achieved.
Duality of interest The author declares that there is no duality of interest associated with this manuscript.

\section{References}

1. Scott DA, Loveman E, McIntyre L, Waugh N (2002) Screening for gestational diabetes: a systematic review and economic evaluation. Health Technol Assess 6:1-161

2. Waugh N, Royle P, Clar C et al (2010) Screening for hyperglycaemia in pregnancy: a rapid update for the national screening committee. Health Technol Assess 14:1-183

3. Metzger BE, Lowe LP, Dyer AR et al (2008) Hyperglycemia and adverse pregnancy outcomes. N Engl J Med 358:1991-2002

4. Crowther CA, Hiller JE, Moss JR, McPhee AJ, Jeffries WS, Robinson JS (2005) Australian carbohydrate intolerance study in pregnant women (ACHOIS) trial group.: Effect of treatment of gestational diabetes mellitus on pregnancy outcomes. N Engl J Med 352:2477-2486

5. Landon MB, Spong CY, Thom E et al (2009) A multicenter, randomized trial of treatment for mild gestational diabetes. N Engl J Med 361:1339-1348

6. Rowan JA, Hague WM, Gao W, Battin MR, Moore MP (2008) Metformin vs insulin for the treatment of gestational diabetes. N Engl J Med 358:2003-2015

7. Langer O, Conway DL, Berkus MD, Xenakis EM, Gonzales O (2000) A comparison of glyburide and insulin in women with gestational diabetes mellitus. N Engl J Med 343:1134-1138

8. Round JA, Jacklin P, Fraser RB, Hughes RG, Mugglestone MA, Holt RI (2010) Screening for gestational diabetes mellitus: costutility of different screening strategies based on a woman's individual risk of disease. Diabetologia doi:10.1007/s00125-0101881-y

9. Sermer M, Naylor CD, Gare DJ et al (1995) Impact of increasing carbohydrate intolerance on maternal-fetal outcomes in 3637 women without gestational diabetes. The Toronto trihospital gestational diabetes project. Am J Obstet Gynecol 173:146-156

10. Sacks DA, Greenspoon JS, Abu-Fadil S, Henry HM, WoldeTsadik G, Yao JF (1995) Toward universal criteria for gestational diabetes: the 75-gram glucose tolerance test in pregnancy. Am J Obstet Gynecol 172:607-614

11. International Association of Diabetes and Pregnancy Study Groups Consensus Panel (2010) International association of diabetes and pregnancy study groups recommendations on the diagnosis and classification of hyperglycemia in pregnancy. Diabetes Care 33:676-82

12. National Institute for Health and Clinical Excellence (2008) Diabetes in pregnancy: management of diabetes and its complications from pre-conception to the postnatal period. NICE clinical guideline 63. NICE, London 